\title{
MULTIPLIERS ON WEIGHTED BESOV \\ SPACES OF ANALYTIC FUNCTIONS
}

\author{
OSCAR BLASCO \\ Departamento de Matemáticas \\ Universidad de Zaragoza
}

\begin{abstract}
We characterize the space of multipliers between certain weighted Besov spaces of analytic functions. This extend and give a new proof of a result of Wojtaszczyk about multipliers between Bergman spaces.
\end{abstract}

\section{INTRODUCTION.}

P. Wojtaszczyk $[\mathrm{W}]$, using certain factorization theorems due to Maurey and Grothendieck, proved the following results:

Let $\alpha>0,0<p \leq 2 \leq q<\infty$ and $\frac{1}{r}=\frac{1}{p}-\frac{1}{q}$.

$$
\left(B_{q}, B_{p}\right)=\left\{\lambda_{n}: \sup _{2^{n} \leq k<2^{n+1}}\left(k^{-1 / r}\left|\lambda_{k}\right|\right) \in l^{r}\right\}
$$

$$
\left(X_{\alpha}, B_{p}\right)=\left\{\lambda_{n}: \sup _{2^{n} \leq k<2^{n+1}}\left(k^{\alpha-1 / p}\left|\lambda_{k}\right|\right) \in l^{p}\right\}
$$

where $B_{p}$ and $X_{\alpha}$ stand for the spaces

$$
\begin{gathered}
B_{p}=\left\{f: D \rightarrow \mathbb{C} \text { analytic }:\left(\int_{D}|f(z)|^{p} d \sigma(z)\right)^{1 / p}<\infty\right\} \text { and } \\
X_{\alpha}=\left\{f: D \rightarrow \mathbb{C} \text { analytic }:|f(z)|=O\left(\frac{1}{(1-|z|)^{\alpha}}\right)\right\} .
\end{gathered}
$$

The main objective of this paper is to extend such results to a much more general situation of general weighted Bergman and Besov spaces. We shall present a proof based simply on Kintchine's inequality for the analogue to (0.1) and then we shall use the previous case combined with some duality arguments to get the analogue to $(0.2)$.

1991 Mathematics Subject Classification. 42A45.

Key words and phrases. Besov spaces, multipliers.

This research has been partially supported by the Spanish DGICYT, Proyecto PS89-0106

Typeset by $\mathcal{A} \mathcal{M S}-\mathrm{TEX}_{\mathrm{E}}$ 
Given be a nondecreasing function on $(0,1)$, say $\rho$, such that $\frac{\rho(t)}{t} \in L^{1}((0,1))$, we denote by $B_{p}(\rho), 0<p<\infty$, the space of analytic function $f$ on the unit disc such that

$$
\|f\|_{p, \rho}=\left(\int_{D} \frac{\rho(1-|z|)}{(1-|z|)}|f(z)|^{p} d \sigma(z)\right)^{1 / p}<\infty .
$$

For certain weights the spaces $B_{p}(\rho)$ have been extensively studied in the literature. They can be regarded as extensions of the classical Bergman spaces $(\rho(t)=t)$. Although the condition appearing in the case $p=1$ and $\rho(t)=t^{1 / q-1}$ for $q<1$ goes back to the work of Hardy and Littlewood (see [HL1, Theorem 3.1], the corresponding spaces were first studied as Banach spaces by P. Duren, B.W. Romberg and A.L. Shield in [DRS] and by A.L. Shields and D.L. Williams [SW] for certain weights. Later J. Shapiro [Sh] considered the spaces, denoted by $A_{\alpha}^{p}$ and called weighted Bergman spaces, for $0<p \leq \infty$ and $\rho(t)=t^{\alpha+1}$.

The extension of $(0.1)$ and (0.2) for weighted Bergman spaces can be achieved from similar arguments as those used by Wojtaszczyk, as it was pointed out in [W], when we deal with weights of the type considered in [SW]. In fact they can be obtained, as we shall prove, not only for these weights but for bit more general weights, defined by Janson $[\mathrm{J}]$, under Dini and $b_{\alpha}$ condition (see definitions below).

In [B1] the author gave a procedure to characterize multipliers acting on $B_{p}(\rho)$ when $0<p \leq 1$ and $\rho$ verifies the previous conditions (see [B1, section 3]). Here we shall complete the cases when $2 \leq p<\infty$, and not only for weighted Bergman spaces but for weighted Besov classes of analytic functions.

For $0<p \leq \infty, 0<q<\infty$, we denote by $H(p, q, \rho)$ the spaces formed by analytic functions on the unit disc $D$ satisfying

$$
\|f\|_{p, q, \rho}=\left(\int_{0}^{1} \frac{\rho(1-r)}{1-r} M_{p}^{q}(f, r) d r\right)^{1 / q}<\infty,
$$

and by $H_{\rho}^{p}$ the spaces of analytic functions such that

$$
M_{p}(f, r)=O\left(\frac{\rho(1-r)}{1-r}\right) \quad(r \rightarrow 1) .
$$

The definition of these classes for the particular case of $\rho(t)=t^{\alpha}$, goes back to Hardy and Littlewood (see [HL1] [HL2]) and they were extensively studied for different reasons and by different authors (see [F1, F2, M, MP1, MP2, S]).

Given two sequence spaces $X, Y$ we denote by $(X, Y)$ the space of multipliers from $X$ to $Y$, that is $(X, Y)=\left\{\left(\lambda_{n}\right):\left(\lambda_{n} a_{n}\right) \in Y\right.$ for every $\left.\left(a_{n}\right) \in X\right\}$.

We identify $H(p, q, \rho)$ and $H_{\rho}^{p}$ with sequence spaces by associating with each analytic function the sequence of its Taylor coefficients. Hence $\lambda$ will stand for either the sequence $\left(\lambda_{n}\right)$ or the function $\lambda(z)=\sum_{n=0}^{\infty} \lambda_{n} z^{n}$.

Theorem 1. Let $0<p_{2} \leq 2 \leq p_{1}<\infty, 0<q_{1}, q_{2}<\infty$, and $\rho_{1}, \rho_{2}$ weight functions verifying Dini and $b_{\alpha_{1}}$ and $b_{\alpha_{2}}$ respectively for some $\alpha_{1}, \alpha_{2}>0$. Let $\frac{1}{r}=\frac{1}{\min \left(q_{1}, q_{2}\right)}-\frac{1}{q_{1}}$. Then

$$
\left(H\left(p_{1}, q_{1}, \rho_{1}\right), H\left(p_{2}, q_{2}, \rho_{2}\right)\right)=\left\{\lambda_{n}: \sup _{2^{n} \leq k<2^{n+1}}\left(\rho_{2}\left(k^{-1}\right)^{1 / q_{2}} \rho_{1}\left(k^{-1}\right)^{-1 / q_{1}}\left|\lambda_{k}\right|\right) \in l^{r}\right\} .
$$


Theorem 2. Let $2 \leq p_{1} \leq \infty, 1<p_{2} \leq 2,1<q_{2}<\infty$ and $\rho_{1}, \rho_{2}$ weight functions verifying Dini and $b_{1}$ and $b_{\alpha_{2}}$ respectively for some $\alpha_{2}>0$. Then

$$
\left(H_{\rho_{1}}^{p_{1}}, H\left(p_{2}, q_{2}, \rho_{2}\right)\right)=\left\{\lambda_{n}: \sup _{2^{n} \leq k<2^{n+1}}\left(k \rho_{2}\left(k^{-1}\right)^{1 / q_{2}} \rho_{1}\left(k^{-1}\right)\left|\lambda_{k}\right|\right) \in l^{q_{2}}\right\} .
$$

The reader is referred to [B2] for these and more results about multipliers when $\rho(t)=t^{\alpha}$.

The key point for these results is that for $p \geq 2$ multipliers on $H(p, q, \rho)$ depend on those on $H(2, q, \rho)$ and this space is isomorphic to $l(2, q)$ which makes them very easy to deal with.Then some duality arguments allow us to get Theorem 2 from Theorem 1.

Throughout the paper all functions $f$ will be analytic on the unit disc and $M_{p}(f, r)$ stands for $\left(\int_{0}^{2 \pi}\left|f\left(r e^{i \theta}\right)\right|^{p} \frac{d \theta}{2 \pi}\right)^{1 / p}$. A weight function $\rho$ will be a nondecreasing function on $(0,1)$ with $\rho\left(0^{+}\right)=0$ such that $\frac{\rho(t)}{t} \in L^{1}((0,1))$ and $C$ will be a numerical constant not necessarily the same in each instance.

\section{$\S 1 . \quad$ Definitions and Lemmas.}

There are several types of weight functions that have been considered to extend those results valid for $\rho(t)=t^{\alpha}$ to more general situations (see [SW, BS, BTS, MP2, J]). We shall be dealing with conditions considered in $[\mathrm{J}]$, which cover the other cases and have been found adequated to many other situations.

Definition 1. A weight function will be a non negative non-decreasing function on $(0,1)$ such that $\rho(t) / t \in L^{1}((0,1))$. $\rho$ is said to be a Dini-weight if

$$
\int_{0}^{s} \frac{\rho(t)}{t} d t \leq C \rho(s)
$$

Given $0<\alpha<\infty, \rho$ is said to be a $b_{\alpha}$-weight, $\rho \in b_{\alpha}$, if

$$
\int_{s}^{1} \frac{\rho(t)}{t^{\alpha+1}} d t \leq C \frac{\rho(s)}{s^{\alpha}}
$$

Remark 1. The main example of a Dini-weight $\rho$ such that $\rho \in b_{\alpha+\varepsilon}$ for any $\varepsilon>0$ is

$$
\rho(t)=C_{\alpha, \beta} t^{\alpha}\left(\log \frac{e}{t}\right)^{\beta} .
$$

Remark 2. It is not difficult to see that if $\rho \in b_{\alpha}$ for some $\alpha>0$, then the Dini condition is equivalent to

$$
\int_{0}^{s} \frac{\rho(t)}{t} d t=O(\rho(s)) \quad(s \rightarrow 0)
$$

It is clear that $b_{\alpha}$ condition can be written

$$
\int_{s}^{1} \frac{\rho(t)}{t^{\alpha+1}} d t \approx \frac{\rho(s)}{s^{\alpha}}
$$

which implies that there is a constant $C<1$ such that

$$
C \rho(2 s) \leq \rho(s) \leq \rho(2 s) \quad\left(0<s<\frac{1}{2}\right) .
$$

Let us include now some results on weights to be used later on. 
Lemma 1. Let $\rho$ be a Dini weight such that $\rho \in b_{\alpha}$. Then

$$
\begin{gathered}
\rho\left(2^{-n}\right) \in l^{1} \text { and } \quad \sum_{m=n}^{\infty} \rho\left(2^{-m}\right)=O\left(\rho\left(2^{-n}\right)\right) \quad(n \rightarrow \infty) \\
\int_{0}^{1} \frac{\rho(1-r)}{(1-r)} r^{n} d r=O\left(\rho\left(\frac{1}{n}\right)\right) \quad(n \rightarrow \infty) \quad(\text { see }[\text { B1, Lemma } 1.1])
\end{gathered}
$$

Proof. Note that from (1.3)

$$
\rho\left(2^{-n}\right) \approx \int_{1-2^{-n}}^{1-2^{-(n+1)}} \frac{\rho(1-r)}{1-r} d r .
$$

Therefore $\rho\left(2^{-n}\right) \in l^{1}$ follows from $\frac{\rho(t)}{t} \in L^{1}((0,1))$ and

$$
\sum_{m=n}^{\infty} \rho\left(2^{-m}\right) \approx \int_{1-2^{-n}}^{1} \frac{\rho(1-r)}{1-r} d r \approx \int_{0}^{2^{-n}} \frac{\rho(t)}{t} d t=O\left(\rho\left(2^{-n}\right)\right) .
$$

Definition 2. Let $0<p \leq \infty, 0<q<\infty$ and $\rho$ a weight function. $H(p, q, \rho)$ will denote the space of analytic functions on the unit disc $D$ satisfying

$$
\|f\|_{p, q, \rho}=\left(\int_{0}^{1} \frac{\rho(1-r)}{1-r} M_{p}^{q}(f, r) d r\right)^{1 / q}<\infty .
$$

Let $\rho$ be a weight function with inf $\frac{\rho(1-r)}{1-r}>0 . H_{\rho}^{p}$ will denote the space of analytic functions on the unit disc $D$ satisfying

$$
M_{p}(f, r)=O\left(\frac{\rho(1-r)}{1-r}\right) .
$$

We define the norm by

$$
\|f\|_{p, \rho}=\inf \left\{C: M_{p}(f, r) \leq C \frac{\rho(1-r)}{1-r}, 0<r<1\right\} .
$$

Definition 3. Let $0<p \leq \infty, 0<q<\infty$ and $\bar{\gamma}=\left(\gamma_{n}\right)$ a sequence of positive real numbers. Denote by $I_{n}=\left\{k \in \mathbb{N}: 2^{n-1} \leq k<2^{n}\right\}$ and $I_{0}=\{0\}$.

$$
l(p, q, \bar{\gamma})=\left\{\left(a_{n}\right) \in \mathbb{C}:\left\|\left(a_{n}\right)\right\|_{p, q, \bar{\gamma}}=\left(\sum_{n}\left(\sum_{k \in I_{n}}\left|a_{n}\right|^{p}\right)^{q / p} \gamma_{n}\right)^{1 / q}<\infty\right\}
$$

We denote by $l(p, q)$, with the obvious modification for $q=\infty$, the case where $\gamma_{n}=1$ for all $n \in \mathbb{N}$.

Let us recall a very useful example of multipliers to be used later on. 
Lemma 2. ([K]) Let $0<p_{1}, q_{1}, p_{2}, q_{2} \leq \infty$ Then

$$
\left(l\left(p_{1}, q_{1}\right), l\left(p_{2}, q_{2}\right)\right)=l(p, q)
$$

where $\frac{1}{p}=\frac{1}{\min \left(p_{1}, p_{2}\right)}-\frac{1}{p_{1}}$ and $\frac{1}{q}=\frac{1}{\min \left(q_{1}, q_{2}\right)}-\frac{1}{q_{1}}$.

Note that Plancherel's theorem implies

$$
\begin{aligned}
\|f\|_{2,2, \rho} & =\left(\int_{0}^{1} \frac{\rho(1-r)}{1-r} \sum_{n=0}^{\infty}\left|a_{n}\right|^{2} r^{2 n} d r\right)^{1 / 2} \\
& =\left(\sum_{n=0}^{\infty}\left|a_{n}\right|^{2} \int_{0}^{1} \frac{\rho(1-r)}{1-r} r^{2 n} d r\right)^{1 / 2} \approx\left(\sum_{n=0}^{\infty}\left|a_{n}\right|^{2} \rho\left(n^{-1}\right)\right)^{1 / 2} .
\end{aligned}
$$

Hence, for $\rho$ verifying (1.3), we have

$$
H(2,2, \rho)=\left\{\lambda_{n}: \lambda_{n} \in l\left(2,2, \rho\left(2^{-n}\right)\right)\right\}
$$

To extend this to other values of $0<q<\infty$ (see [MP1, S] for the case $\rho(t)=t^{\alpha}$ ) we shall use the following lemma.

Lemma 3. Let $0<q<\infty, \rho$ a Dini weight with $\rho \in b_{\alpha}$ for some $\alpha>0$ and $\alpha_{n} \geq 0$.Then

$$
\int_{0}^{1} \frac{\rho(1-r)}{1-r}\left(\sum_{n=0}^{\infty} \alpha_{n} r^{n}\right)^{q} d r \approx \sum_{n=0}^{\infty}\left(\sum_{k \in I_{n}} \alpha_{k}\right)^{q} \rho\left(2^{-n}\right)
$$

Proof.

$$
\begin{aligned}
\int_{0}^{1} \frac{\rho(1-r)}{1-r}\left(\sum_{n=0}^{\infty} \alpha_{n} r^{n}\right)^{q} d r & =\sum_{n=0}^{\infty} \int_{1-2^{-n}}^{1-2^{-(n+1)}} \frac{\rho(1-r)}{1-r}\left(\sum_{n=0}^{\infty} \alpha_{n} r^{n}\right)^{q} d r \\
& \geq C \sum_{n=0}^{\infty} \int_{1-2^{-n}}^{1-2^{-(n+1)}} \frac{\rho(1-r)}{1-r}\left(\sum_{k \in I_{n}} \alpha_{k}\right)^{q} r^{2^{n} q} d r \\
& \geq C \sum_{n=0}^{\infty}\left(\sum_{k \in I_{n}} \alpha_{k}\right)^{q} \rho\left(2^{-n}\right) .
\end{aligned}
$$

Let us now show the converse inequality. Assume first $0<q \leq 1$.

$$
\begin{aligned}
\int_{0}^{1} \frac{\rho(1-r)}{1-r}\left(\sum_{n=0}^{\infty} \alpha_{n} r^{n}\right)^{q} d r & \leq \int_{0}^{1} \frac{\rho(1-r)}{1-r}\left(\sum_{n=0}^{\infty}\left(\sum_{k \in I_{n}} \alpha_{k}\right) r^{2^{n}-1}\right)^{q} d r \\
& \leq \int_{0}^{1} \frac{\rho(1-r)}{1-r}\left(\sum_{n=0}^{\infty}\left(\sum_{k \in I_{n}} \alpha_{k}\right)^{q} r^{\left(2^{n}-1\right) q}\right) d r \\
& \leq \sum_{n=0}^{\infty}\left(\sum_{k \in I_{n}} \alpha_{k}\right)^{q} \int_{0}^{1} \frac{\rho(1-r)}{1-r} r^{\left(2^{n}-1\right) q} d r \\
& \leq C \sum_{n=0}^{\infty}\left(\sum_{k \in I_{n}} \alpha_{k}\right)^{q} \rho\left(2^{-n}\right)
\end{aligned}
$$


where the last inequality follows from (1.5).

Let us then asumme $q>1$.

$$
\begin{aligned}
\int_{0}^{1} \frac{\rho(1-r)}{1-r}\left(\sum_{n=0}^{\infty} \alpha_{n} r^{n}\right)^{q} d r & =\sum_{n=0}^{\infty} \int_{1-2^{-n}}^{1-2^{-(n+1)}} \frac{\rho(1-r)}{1-r}\left(\sum_{n=0}^{\infty} \alpha_{n} r^{n}\right)^{q} d r \\
& \leq C \sum_{n=0}^{\infty} \int_{1-2^{-n}}^{1-2^{-(n+1)}} \frac{\rho(1-r)}{1-r}\left(\sum_{m=0}^{n} \sum_{k \in I_{m}} \alpha_{k}\right)^{q} d r \\
& +C \sum_{n=0}^{\infty} \int_{1-2^{-n}}^{1-2^{-(n+1)}} \frac{\rho(1-r)}{1-r}\left(\sum_{m=n+1}^{\infty}\left(\sum_{k \in I_{m}} \alpha_{k}\right) r^{2^{m-1}}\right)^{q} d r \\
& =(I)+(I I) .
\end{aligned}
$$

In order to estimate $(I)$ note that for any sequence $\beta_{n} \geq 0$ we have

$$
\sum_{n=0}^{\infty}\left(\sum_{m=0}^{n} \beta_{m}\right)^{q} \rho\left(2^{-n}\right) \leq C \sum_{n=0}^{\infty} \beta_{n}^{q} \rho\left(2^{-n}\right)
$$

Indeed, put $\gamma_{n+1}=\sum_{m=0}^{n} \beta_{m}, \gamma_{0}=0$ and $\mu(A)=\sum_{n \in A} \rho\left(2^{-n}\right)$, then

$$
\begin{aligned}
\sum_{n=0}^{\infty}\left(\sum_{m=0}^{n} \beta_{m}\right)^{q} \rho\left(2^{-n}\right) & =q \int_{0}^{\infty} \lambda^{q-1} \mu\left(\left\{n: \sum_{m=0}^{n} \beta_{m}>\lambda\right\}\right) d \lambda \\
& =q \sum_{n=0}^{\infty} \int_{\gamma_{n}}^{\gamma_{n+1}} \lambda^{q-1} \mu\left(\left\{k: \sum_{m=0}^{k} \beta_{m}>\lambda\right\}\right) d \lambda \\
& \leq C \sum_{n=0}^{\infty}\left(\sum_{m=0}^{n} \beta_{m}\right)^{q-1}\left(\sum_{m=n}^{\infty} \rho\left(2^{-m}\right)\right)\left(\gamma_{n+1}-\gamma_{n}\right) \\
& \leq C \sum_{n=0}^{\infty}\left(\sum_{m=0}^{n} \beta_{m}\right)^{q-1} \beta_{n} \rho\left(2^{-n}\right) \\
& \leq C\left(\sum_{n=0}^{\infty}\left(\sum_{m=0}^{n} \beta_{m}\right)^{q} \rho\left(2^{-n}\right)\right)^{1 / q^{\prime}}\left(\sum_{n=0}^{\infty} \beta_{m}^{q} \rho\left(2^{-n}\right)\right)^{1 / q}
\end{aligned}
$$

This gives (1.7), what allows us to get by writing $\beta_{n}=\sum_{k \in I_{n}} \alpha_{k}$

$$
(I) \leq C \sum_{n=0}^{\infty} \sum_{m=0}^{n}\left(\sum_{k \in I_{m}} \alpha_{k}\right)^{q} \rho\left(2^{-n}\right) \leq C \sum_{n=0}^{\infty}\left(\sum_{k \in I_{n}} \alpha_{k}\right)^{q} \rho\left(2^{-n}\right) .
$$

In order to estimate $(I I)$, first observe that for any $\beta_{n} \geq 0$

$$
\begin{aligned}
\left(\sum_{m=n+1}^{\infty} \beta_{m} r^{2^{m-1}}\right)^{q} & \leq\left(\sum_{m=n+1}^{\infty} 2^{-m \alpha} \beta_{m}^{q}\right)\left(\sum_{m=n+1}^{\infty} 2^{m \alpha\left(q^{\prime}-1\right)} r^{\left(2^{m-1}\right) q^{\prime}}\right)^{q-1} \\
& \leq C\left(\sum_{m=n+1}^{\infty} 2^{-m \alpha} \beta_{m}^{q}\right)\left(\sum_{m=2^{n}}^{\infty} m^{\alpha\left(q^{\prime}-1\right)-1} r^{m q^{\prime}}\right)^{q-1} \\
& \leq C\left(\sum_{m=n+1}^{\infty} 2^{-m \alpha} \beta_{m}^{q}\right) \frac{1}{(1-r)^{\alpha}}
\end{aligned}
$$


Hence

$$
\begin{aligned}
(I I) & \leq C \sum_{n=0}^{\infty} \int_{1-2^{-n}}^{1-2^{-(n+1)}} \frac{\rho(1-r)}{1-r}\left(\sum_{m=n+1}^{\infty}\left(\sum_{k \in I_{m}} \alpha_{k}\right) r^{2^{m-1}}\right)^{q} d r \\
& \leq C \sum_{n=0}^{\infty}\left(\sum_{m=n+1}^{\infty} 2^{-m \alpha}\left(\sum_{k \in I_{m}} \alpha_{k}\right)^{q}\right) \int_{1-2^{-n}}^{1-2^{-(n+1)}} \frac{\rho(1-r)}{(1-r)^{\alpha+1}} d r \\
& \leq C \sum_{m=0}^{\infty}\left(\sum_{k \in I_{m}} \alpha_{k}\right)^{q} 2^{-m \alpha} \sum_{n=0}^{m} \int_{1-2^{-n}}^{1-2^{-(n+1)}} \frac{\rho(1-r)}{(1-r)^{\alpha+1}} d r \\
& =C \sum_{m=0}^{\infty}\left(\sum_{k \in I_{m}} \alpha_{k}\right)^{q} 2^{-m \alpha} \int_{0}^{1-2^{-(m+1)}} \frac{\rho(1-r)}{(1-r)^{\alpha+1}} d r \\
& \leq C \sum_{m=0}^{\infty}\left(\sum_{k \in I_{m}} \alpha_{k}\right)^{q} \rho\left(2^{-m}\right) .
\end{aligned}
$$

Corollary 1. Let $0<q<\infty, \rho$ a Dini weight, $\rho \in b_{\alpha}$ and $f(z)=\sum_{n=1}^{\infty} a_{n} z^{n}$. Then

$$
f \in H(2, q, \rho) \text { if and only if } a_{n} \in l\left(2, q, \rho\left(2^{-n}\right)\right) \text {. }
$$

Corollary 2. Let $1<q<\infty, \frac{1}{q}+\frac{1}{q^{\prime}}=1, \rho$ a Dini weight and $\rho \in b_{\alpha}$. Then

$$
(H(2, q, \rho))^{*}=H\left(2, q^{\prime}, \rho\right) \text {. }
$$

under the duality pair $\langle f, g\rangle=\sum_{n=0}^{\infty} a_{n} \bar{b}_{n} \rho\left(\frac{1}{n}\right)$.

Remark 3. For other values of $1 \leq p \leq \infty$ using (1.5)

$$
\begin{aligned}
\sum_{n=0}^{\infty} a_{n} \bar{b}_{n} \rho\left(n^{-1}\right) & \approx \int_{0}^{1} \frac{\rho(1-r)}{1-r} \sum_{n=0}^{\infty} a_{n} \bar{b}_{n} r^{n} d r \\
& =\int_{0}^{1} \frac{\rho(1-r)}{1-r} \int_{0}^{2 \pi} f\left(r e^{i t}\right) \bar{g}\left(r e^{i t}\right) \frac{d t}{2 \pi} \\
& \leq C \int_{0}^{1} \frac{\rho(1-r)}{1-r} M_{p}(f, r) M_{p^{\prime}}(g, r) d r \\
& \leq C\|f\|_{p, q, \rho}\|g\|_{p^{\prime}, q^{\prime}, \rho}
\end{aligned}
$$

This shows that for $1 \leq p \leq \infty$ and $1<q<\infty$

$$
H\left(p^{\prime}, q^{\prime}, \rho\right) \subset(H(p, q, \rho))^{*} .
$$

Actually for the case $1<p<\infty$ it can be shown that $H\left(p^{\prime}, q^{\prime}, \rho\right)$ is the dual of $H(p, q, \rho)$. Altough we shall not need the duality result the reader is referred to $[\mathrm{S}]$ for a proof that can be easily extended to the weighted case.

Let us finally mention the duality for the case $q=1$. 
Theorem A. (See [BS, Theorem 3.2]. Let $1 \leq p<\infty$ and $\rho$ a Dini weight such that $\rho \in b_{1}$. Then

$$
(H(p, 1, \rho))^{*}=H_{\rho}^{p^{\prime}}
$$

under the pairing $<f, g>_{\alpha}=\lim _{r \rightarrow 1} \sum_{n=0}^{\infty}(n+1)^{-1} a_{n} b_{n} r^{n}$.

Remark 4. This can be extended to weights having $b_{\alpha}$ condition by means of fractional derivatives (see $[\mathrm{B} 2])$.

\section{$\S 4$ The Theorems And Their Proofs.}

Next result follows easily from Kintchine's inequality.

Lemma 4. Let $0<p, q<\infty$. Let $f(z)=\sum_{n=0}^{\infty} a_{n} z^{n}$. Let us denote by $f_{t}(z)=$ $\sum_{n=0}^{\infty} r_{n}(t) a_{n} z^{n}$ where $r_{n}$ are the Rademacher functions.

$$
M_{2}^{q}(f, r) \approx \int_{0}^{1} M_{p}^{q}\left(f_{t}, r\right) d t
$$

Proposition 1. Let $0<p_{2} \leq 2 \leq p_{1}<\infty, 0<q_{1}, q_{2}<\infty$ and $\rho_{1}, \rho_{2}$ weight functions. Then

$$
\left(\left(H\left(p_{1}, q_{1}, \rho_{1}\right), H\left(p_{2}, q_{2}, \rho_{2}\right)\right)=\left(\left(H\left(2, q_{1}, \rho_{1}\right), H\left(2, q_{2}, \rho_{2}\right)\right) .\right.\right.
$$

Proof. It is immediate that

$$
\left(( H ( 2 , q _ { 1 } , \rho _ { 1 } ) , H ( 2 , q _ { 2 } , \rho _ { 2 } ) ) \subset \left(\left(H\left(p_{1}, q_{1}, \rho_{1}\right), H\left(p_{2}, q_{2}, \rho_{2}\right)\right) .\right.\right.
$$

Take now $\lambda_{n} \in\left(\left(H\left(p_{1}, q_{1}, \rho_{1}\right), H\left(p_{2}, q_{2}, \rho_{2}\right)\right)\right.$ and $f \in H\left(p_{1}, q_{1}, \rho_{1}\right)$. Denote by $(f * \lambda)(z)=\sum_{n=0}^{\infty} a_{n} \lambda_{n} z^{n}$ and $(f * \lambda)_{t}(z)=\sum_{n=0}^{\infty} r_{n}(t) a_{n} \lambda_{n} z^{n}$ where $r_{n}(t)$ stand for the Rademacher functions.

An application of (2.1) and Fubini's Theorem give

$$
\begin{aligned}
\int_{0}^{1} \frac{\rho_{2}(1-r)}{1-r} M_{2}^{q_{2}}(f * \lambda, r) d r & \leq C \int_{0}^{1} \frac{\rho_{2}(1-r)}{1-r} \int_{0}^{1} M_{p_{2}}^{q_{2}}\left((f * \lambda)_{t}, r\right) d t d r \\
& =C \int_{0}^{1} \int_{0}^{1} \frac{\rho_{2}(1-r)}{1-r} M_{p_{2}}^{q_{2}}\left((f * \lambda)_{t}, r\right) d r d t \\
& \leq C \int_{0}^{1}\left(\int_{0}^{1} \frac{\rho_{1}(1-r)}{1-r} M_{p_{1}}^{q_{1}}\left(f_{t}, r\right) d r\right)^{q_{2} / q_{1}} d t=A
\end{aligned}
$$

Now if $q_{2} \leq q_{1}$ then from Fubini and (2.1) again

$$
\begin{aligned}
A & \leq\left(\int_{0}^{1} \int_{0}^{1} \frac{\rho_{1}(1-r)}{1-r} M_{p_{1}}^{q_{1}}\left(f_{t}, r\right) d r d t\right)^{q_{2} / q_{1}} \\
& \leq\left(\int_{0}^{1} \frac{\rho_{1}(1-r)}{1-r} \int_{0}^{1} M_{p_{1}}^{q_{1}}\left(f_{t}, r\right) d t d r\right)^{q_{2} / q_{1}} \\
& \leq C\left(\int_{0}^{1} \frac{\rho_{1}(1-r)}{1-r} M_{2}^{q_{1}}(f, r) d r\right)^{q_{2} / q_{1}}
\end{aligned}
$$


If $q_{2}>q_{1}$ put $s=\frac{q_{2}}{q_{1}}$ and apply duality

$$
\begin{aligned}
A & =\sup _{\|h\|_{s^{\prime}}=1}\left(\int_{0}^{1}\left(\int_{0}^{1} \frac{\rho_{1}(1-r)}{1-r} M_{p_{1}}^{q_{1}}\left(f_{t}, r\right) d r\right) h(t) d t\right)^{q_{2} / q_{1}} \\
& =\sup _{\|h\|_{s^{\prime}=1}}\left(\int_{0}^{1}\left(\int_{0}^{1} M_{p_{1}}^{q_{1}}\left(f_{t}, r\right) h(t) d t\right) \frac{\rho_{1}(1-r)}{1-r} d r\right)^{q_{2} / q_{1}} \\
& \leq\left(\int_{0}^{1}\left(\int_{0}^{1} M_{p_{1}}^{q_{2}}\left(f_{t}, r\right) d t\right)^{q_{1} / q_{2}} \frac{\rho(1-r)}{1-r} d r\right)^{q_{2} / q_{1}} \\
& \leq C\left(\int_{0}^{1} \frac{\rho(1-r)}{1-r} M_{2}^{q_{1}}(f, r) d r\right)^{q_{2} / q_{1}} \cdot
\end{aligned}
$$

Theorem 1. Let $0<p_{2} \leq 2 \leq p_{1}<\infty, 0<q_{1}, q_{2}<\infty$, and $\rho_{1}, \rho_{2}$ weight functions verifying Dini and $b_{\alpha_{1}}$ and $b_{\alpha_{2}}$ respectively for some $\alpha_{1}, \alpha_{2}>0$. Let $\frac{1}{r}=\frac{1}{\min \left(q_{1}, q_{2}\right)}-\frac{1}{q_{1}}$. Then

$$
\left(H\left(p_{1}, q_{1}, \rho_{1}\right), H\left(p_{2}, q_{2}, \rho_{2}\right)\right)=\left\{\lambda_{n}: \sup _{2^{n} \leq k<2^{n+1}}\left(\rho_{2}\left(k^{-1}\right)^{1 / q_{2}} \rho_{1}\left(k^{-1}\right)^{-1 / q_{1}}\left|\lambda_{k}\right|\right) \in l^{r}\right\} .
$$

Proof. Using Proposition 1 and Corollary 1 we simply have to find

$$
\left(l\left(2, q_{1}, \rho_{1}\left(2^{-n}\right)\right), l\left(2, q_{2}, \rho_{2}\left(2^{-n}\right)\right)\right) .
$$

Note that $\left(\lambda_{n}\right) \in\left(l\left(2, q_{1}, \rho_{1}\left(2^{-n}\right)\right), l\left(2, q_{2}, \rho_{2}\left(2^{-n}\right)\right)\right)$ is equivalent to

$$
\rho_{2}\left(n^{-1}\right)^{1 / q_{2}} \lambda_{n} \rho_{1}\left(n^{-1}\right)^{-1 / q_{1}} \in\left(l\left(2, q_{1}\right), l\left(2, q_{2}\right)\right) .
$$

Hence the proof is completed by invoking Lemma 2.

Lemma 5. (see [B1, Lemma 5.1 and 5.2]) Let $\rho$ be a Dini weight and $\rho(t) \in b_{\alpha}$ for some $\alpha>0$. Let $\left(\alpha_{n}\right) \geq 0$. Then

$$
\sum_{n=0}^{\infty} \alpha_{n} r^{n}=O\left(\frac{\rho(1-r)}{(1-r)^{\alpha}}\right) \quad \text { if and only if } \quad \sum_{m \in I_{n}} n^{-\alpha} \alpha_{n}=O\left(\rho\left(2^{-n}\right)\right) .
$$

Theorem 2. Let $2 \leq p_{1} \leq \infty, 1<p_{2} \leq 2,1<q_{2}<\infty$ and $\rho_{1}, \rho_{2}$ weight functions verifying Dini and $b_{1}$ and $b_{\alpha_{2}}$ respectively for some $\alpha_{2}>0$. Then

$$
\left(H_{\rho_{1}}^{p_{1}}, H\left(p_{2}, q_{2}, \rho_{2}\right)\right)=\left\{\lambda_{n}: \sup _{2^{n} \leq k<2^{n+1}}\left(k \rho_{2}\left(k^{-1}\right)^{1 / q_{2}} \rho_{1}\left(k^{-1}\right)\left|\lambda_{k}\right|\right) \in l^{q_{2}}\right\} .
$$

Proof. It is clear that $\left(H_{\rho_{1}}^{2}, H\left(2, q_{2}, \rho_{2}\right)\right) \subset\left(H_{\rho_{1}}^{p_{1}}, H\left(p_{2}, q_{2}, \rho_{2}\right)\right)$. 
Let $\left(\lambda_{n}\right) \in\left(H_{\rho_{1}}^{p_{1}}, H\left(p_{2}, q_{2}, \rho_{2}\right)\right)$. It is not hard to see from duality (see (1.8) and Theorem A) that then

$$
(n+1) \lambda_{n} \rho_{2}\left(n^{-1}\right) \in\left(H\left(p_{2}^{\prime}, q_{2}^{\prime}, \rho_{2}\right), H\left(p_{1}^{\prime}, 1, \rho_{1}\right)\right) .
$$

Using Proposition 1 and duality again this implies $\left(\lambda_{n}\right) \in\left(H_{\rho_{1}}^{2}, H\left(2, q_{2}, \rho_{2}\right)\right)$, which, from Corollary 1 and Lemma 5, is equivalent to

$$
\rho_{2}\left(n^{-1}\right)^{1 / q_{2}} \lambda_{n} \rho_{1}\left(n^{-1}\right)(n+1) \in\left(l(2, \infty), l\left(2, q_{1}\right)\right) .
$$

The proof is again finished from invoking Lemma 2.

\section{REFERENCES}

[AS] J.M. Anderson, A.L. Shields, Coefficient multipliers on Bloch functions, Trans. Amer. Math. Soc. 224 (1976), 256-265.

[BST] G. Bennett, D.A. Stegenga, R.M. Timoney, Coefficients of Bloch and Lipschitz functions, Illinois J. of Math. 25 (1981), 520-531.

[B1] O. Blasco, Operators on weighted Bergman spaces and applications, Duke Math. J. (to appear).

[B2] O. Blasco, Multipliers on spaces of analytic functions (to appear).

[BS] O. Blasco, G.S. de Souza, Spaces of analytic functions on the disc where the growth of $M_{p}(F, r)$ depends on a weight, J. Math. Anal. and Appl. 147 (1990), 580-598.

[BlS] S. Bloom, G.S. de Souza, Atomic decomposition of generalized Lipschitz spaces, Illinois J. Math. 33 (1989), 181-189.

[D] P. Duren, Theory of $H_{p}$-spaces (1970), Academic Press, New York.

[DS1] P. Duren, A.L. Shields, Coefficient multipliers of $H^{p}$ and $B^{p}$ spaces, Pacific J. Math. 32 (1970), 69-78.

[DS2] P. Duren, A.L. Shields, Properties of $H^{p}(0<p<1)$ and its containing Banach space, Trans. Amer. Math. Soc. 141 (1969), 255-262

[DRS] P.L. Duren, B.W. Romberg, A.L. Shields, Linear functionals on $H_{p}$-spaces with $0<p<1$, J. Reigne Angew. Math. 238 (1969), 32-60.

[F1] T.M. Flett, The dual of an inequality of Hardy and Littlewood and some related inequalities, J. Math. Anal. and Appl. 38 (1972), 746-765.

[F2] T.M. Flett, Lipschitz spaces of functions on the circle and the disc, J. Math. Anal. and Appl. 39 (1972), 125-158.

[F3] T.M. Flett, On the rate of growth of mean valeus of holomorphic and harmonic functions, Proc. London Math. Soc. 20 (1970), 749-768.

[HL1] G.H. Hardy, J.E. Littlewood, Some properties of fractional integrals II, Math. Z. 34 (1932), 403-439.

[HL2] G.H. Hardy, J.E. Littlewood, Theorems concerning mean values of analytic or harmonic functions, Quart. J. Math. 12 (1941), 221-256.

[K] C. N. Kellogg, An extension of the Hausdorff-Young Theorem, Michigan. Math. J. 18 (1971), 121-127.

[J1] S. Janson, Generalization on Lipschitz spaces and applications to Hardy spaces and bounded mean oscillation, Duke Math. J. 47 (1980), 959-982.

[M] M. Marzuq, Linear functionals on some weighted Bergman spaces, Bull. Austral. Math. Soc. 42 (1990), 417-425.

[MP1] M. Mateljevic, M. Pavlovic, $L^{p}$ behaviour of power series with positive coefficients and Hardy spaces, Proc. Amer. Math. Soc. 87 (1983), 309-316.

[MP2] M. Mateljevic, M. Pavlovic, $L^{p}$ behaviour of the integral means of analytic functions, Studia Math. 77 (1984), 219-237. 
[M] A. Matheson, A Multipliers theorem for analytic functions of slow mean growth, Proc. Amer. Math. Soc. 77 (1979), 53-57.

[S] W. Sledd, Some results about spaces of analytic functions introduced by Hardy and Littlewood, J. London Math. Soc. 2 (1974), 328-336.

[Sh] J.H. Shapiro, Mackey topologies, reproducing kernels and diagonal maps on the Hardy and Bergman spaces, Duke Math. J. 43 (1976), 187-202.

[SW] A.L.Shields, D.L. Williams, Bounded projections, duality and multipliers in spaces of analytic functions, Trans. Amer. Math. Soc. 162, (1971), 287-302.

[W] P. Wojtaszcyk, Multipliers into Bergman spaces and Nevalinna class, Canad. Math. Bull. 33 (1990), 151-161.

[Z] K. Zhu, Operator theory in function spaces, Marcel Dekker, Inc., New York, 1990.

Departamento de Matemáticas, Universidad de Zaragoza, 50009 Zaragoza, Spain. Current address:

E-mail address:Blasco@cc.unizar.es 